\title{
Reaction of Indian Chicks to Klebsiella Pneumoniae Strain Borkar, a Plant Pathogen Causing Pneumonia in Plants
}

\author{
S. G. Borkar and T. S. Ajayasree
}

\section{ABSTRACT}

Klebsiella pneumoniae is emerging as an important bacterial plant pathogen in Asia region particularly in China and India. The Chinese strain of Klebsiella pneumoniae causing top rot of maize is reported to be pathogenic on mice also. The Indian strain of Klebsiella pneumoniae causing root bark necrosis and wilt in pomegranate is found non- pathogenic on Indian bird chicken, thereby indicating it to be plant host specific.

Keywords: Klebsiella pneumoniae, plant pathogenic bacteria, reaction, Indian bird chicken.

\author{
Published Online: July 24, 2020 \\ ISSN: $2684-446 \mathrm{X}$ \\ DOI :10.24018/ejgeo.2020.1.4.26 \\ S. G. Borkar \\ Vasantrao Naik Marathwada \\ Agriculture University, India. \\ T.S. Ajayasree * \\ Mahatma Phule Agriculture University, \\ India. \\ (e-mail: ajayasree128@gmail.com) \\ *Corresponding Author
}

The bacteria Klebsiella pneumoniae is a serious human pathogen causing respiratory tract infection, loss of breathing leading to pneumonia and death of patient [9]. The most common symptoms of bacterial pneumonia are a cough with thick yellow, green, or blood tinged mucus, stabbing chest pain that worsen when coughing or breathing, sudden onset of chills severe enough to shake the human body, fever of $102-105^{\circ} \mathrm{F}$ or above. Klebsiella pneumoniae can progress into severe bacterial infection leading to pneumonia, blood stream infection, wound infection, urinary tract infection and meningitis.

Klebsiella infection refers to several different types of healthcare associated infections that are all caused by Klebsiella bacteria. To get Klebsiella infection, a person must be exposed to bacteria. For example Klebsiella must enter the respiratory (breathing) tract to cause pneumonia, or the blood to cause blood stream infection [3], [13].

This bacterial pathogen is also reported to cause infection in animals [11], [12], [4], [8] and birds also [6], [5], [10]. Immuno-deficient animals, especially those with severe immune-deficiencies or defect in immune system, may develop clinical disease associated with Klebsiella infection.

An outbreak of Klebsiella pneumonia infection in dogs with severe enteritis and septicaemia was reported by [11]. The bacterium causes pneumonia in guinea pigs of both sexes and all ages although there are few reports of natural infection of this animal species with this organism [12]. Klebsiella pneumonia subsp. pneumoniae is a cause of sudden death of pre-weaned pigs due to septicaemia with a seasonal occurrence between May and Sept [4]. Nodia Wohlwend et.al [8] isolated third generation cephalosporin resistant
Klebsiella pneumonia isolates from humans and companion animals in Switzerland. Characterization of third generation cephalosporin resistant Klebsiella pneumonia isolates originating mainly from one human hospital $(n=22)$ and one companion animal hospital $(n=25)$ in Bern, Switzerland revealed the absence of epidemiological links between human and animal isolates. Human infection were not associated with the spread of any specific clone, while the majority of animal infection was due to Klebsiella pneumonia sequence type 11 isolates producing plasmidic DHA Ampc. This clonal dissemination within the veterinary hospital emphasizes the need for effective infection control practices.

Systemic infection are more common in birds but local infection involving the upper respiratory tract, skin, oral cavity and crop may occur, especially in psittacines [6]. In adverse situation, Klebsiella pneumoniae can cause kidney failure, lung infection and encephalitis in birds. Dashe, Y. G et.al [5] isolated Klebsiella pneumoniae from birds affected by natural outbreak of highly pathogenic avian influenza (H5N1) in Nigeria. Klebsiella pneumoniae was isolated from 9 samples (1.5\% samples). The pathogen was isolated from liver, lung and trachea of commercial layer and turkeys. During the HPAI outbreaks, Klebsiella pneumoniae was isolated from 9 different flocks of 21805 birds, with a mortality rate of $7.3 \%$ and proportionate mortality rate of $2.5 \%$. The bacterium was not isolated from H5N1 free flocks which served as control. The result of this study indicated that Klebsiella pneumonia may have acted as secondary pathogen to aggravate the clinical signs of $\mathrm{H} 5 \mathrm{~N} 1$ outbreaks that occurred in Nigeria. An outbreak of yolk sac infection and dead-in-shell mortality in common canary caused by Klebsiella pneumonia was reported by [10]. Yolk sac 
infection and dead in shell mortality caused by enterobacteriaceae in birds are not a rare phenomenon, however, there are only few reports indicating the association between these conditions and Klebsiella spp among canary chicks. There have been reports of high mortality among 1 to 3 days old canary chicks in indoor flocks of canaries. Klebsiella pneumonia was isolated as causal agent.

In 2016, the bacterium Klebsiella pneumonia was reported to infect maize plant in China causing bacterial top rot disease of maize in Yunnan province, China and the bacterium was also found pathogenic on mice [7]. In the same year, in India the bacterium Klebsiella pneumonia was found to infect the pomegranate plant causing root bark necrosis and wilt of the infected plants [1]. The Klebsiella pneumoniae infecting pomegranate was identified as Klebsiella pneumoniae strain Borkar (NCBI Gene accession number KY 941097.1) based on 16S rRNA partial gene sequence. Further studies on the plant host range of this bacterium indicated that this bacterial strain causes pneumonia type symptoms in plant seedling of tomato, chilli and eggplant i.e. the bacteria block the root respiration and thereby causes the wilting and death of the plant [2]. Besides the plant species, whether this Klebsiella pneumoniae strain infect and cause disease in other living being was a matter of great concern. Therefore, the effect of this bacterial strain was first studied on Indian young bird chicks (Fig. 1) as these are often found to feed on the leftovers in the farm backyards where the diseased plant material is left by the farmers for its decomposition.

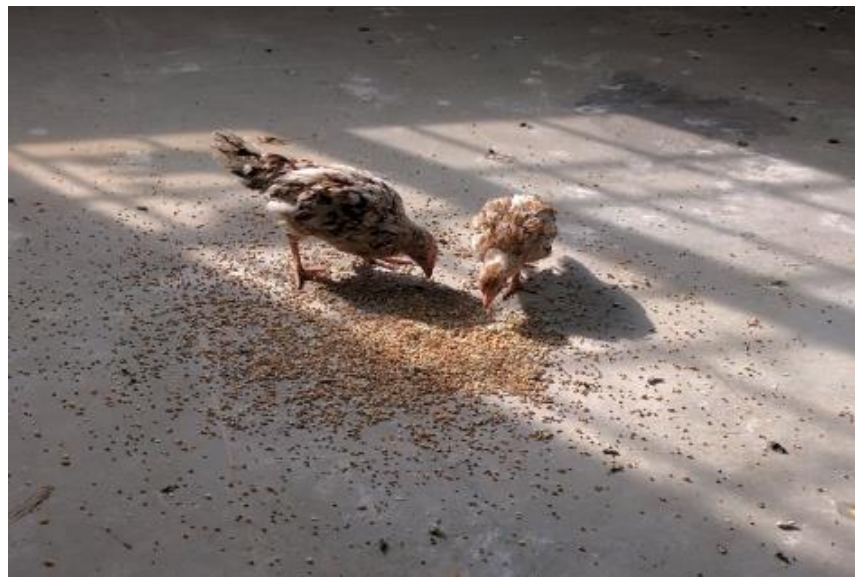

Fig.1. The young bird chicks under experimentation.

The experimentation on the flock of 25 young bird chicks of two month old was carried out under laboratory condition where these chicks were nurtured. The fresh bacterial culture of Klebsiella pneumoniae strain Borkar was prepared from the mother culture strain by sub-culturing. The bacterial suspension was prepared from this young bacterial culture by adding distilled sterile water and $4 \mathrm{ml}$ of this suspension $\left(10^{8}\right.$ $\mathrm{cfu} / \mathrm{ml}$ ) was orally fed to the test experimental bird chicks through their beak by using dropper. Mixed grains of sorghum, pearl-millet, rice and wheat treated with Klebsiella pneumoniae strain (the 50 grams mixed grains were soaked in bacterial suspension for 15 minutes) was fed to the experimental chicks for a day. A chick without oral dose and Klebsiella pneumoniae treated grain served as control. After a day of Klebsiella pneumoniae experimental treatment, regular feed and water was given to the chicks. Daily observations in respect of pneumonia symptoms like fever in the bird, loss of breathing, uneasiness, loss of appetite and ultimate death was taken up to two months.

No symptoms of any kind were observed in the Klebsiella pneumoniae treated experimental chicks. The treated chick did not develop fever; neither had breathing problem nor loss of appetite during the observation period of two months and remained healthy. This indicated that the Klebsiella pneumoniae strain Borkar was non-pathogenic to bird Indian chicks and was only a plant pathogenic bacteria.

The plant pathogenic bacterial nature of Klebsiella pneumoniae strain Borkar was quickly determine by inoculation of the bacterial suspension in potato leaves for the development of hypersensitive reaction. The Klebsiella pneumoniae strain Borkar injected potato leaves developed browning reaction on $2^{\text {nd }}$ day (Fig. 2) with subsequent cracking of injected portion and shot hole symptoms developed on $7^{\text {th }}$ day indicating that the bacterium was plant pathogenic.

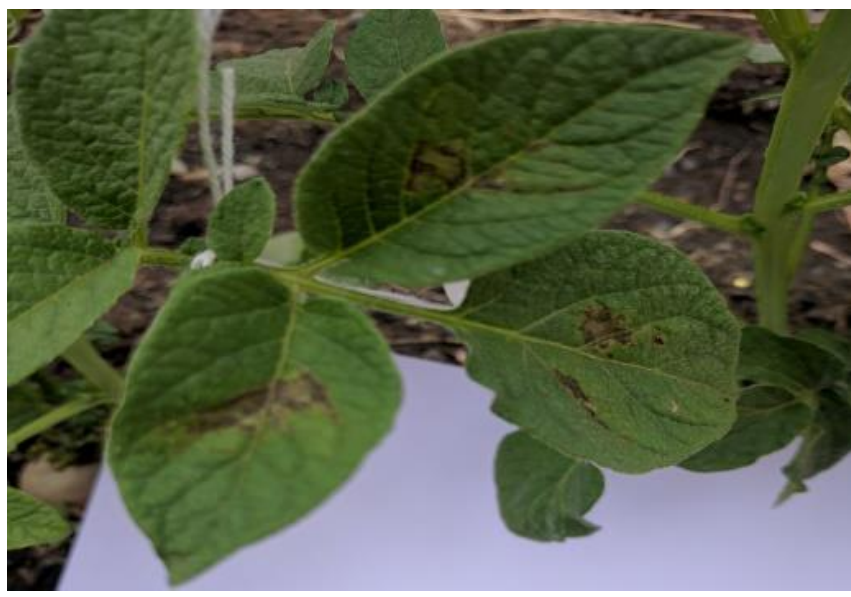

Fig 2. Reaction of Klebsiella pneumoniae on potato leaves.

The Klebsiella pneumoniae strain Borkar is a plant pathogen causing pneumonia in plant but not in the birds particularly the Indian chicks. The genus Klebsiella can now be included in list of plant pathogenic bacterial genus.

\section{REFERENCES}

[1] Ajayasree, T. S. and Borkar, S. G. 2018a. Bacterial root bark necrosis and wilt of pomegranate, hereto a new disease. Journal of Applied Biotechnology \& Bioengineering. 5(6):329-332.

[2] Ajayasree, T. S. and Borkar, S. G. 2018b. Pathogenic potentiality of the bacterium of Klebsiella pneumoniae strain Borkar on different plants. Journal of Applied Biotechnology \& Bioengineering. 5(4):233-235.

[3] Anonomous. 2012. Klebsiella pneumoniae in healthcare setting. Centres for disease control and prevention.

[4] APHA. 2016.Pig expert Group. Apha.defra.gov.uk/documents/surveillance/diseases/klebsiellavet.pdf.

[5] Dashe, Y G., H M Kazeem., P A Abdu., M Bello., M Odugbo. 2008. Klebsiella pneumonia isolates from birds affected by natural outbreak of highly pathogenic avian influenza (H5N1) in Nigeria. Sokoto Journal of Veterinary Science. 7(2):59-61.

[6] Gerlach, H. 1994. Bacteria. In B.W. Ritchie, G.J. Harrlson and L.R. Harrlson. Avian medicine: Principles and application (pp 949-983). Lake Worth, Fl: Wingers publishing.

[7] Huang min, Li Lin, Yi-xin Wu, Ho Honhing, Peng-fei HE, Guo-Zhi LI, Peng-bo HE, Guo-ru Xiong, Yuan Yuan and Yue-qiu HE. 2016. 
Pathogenicity of Klebsiella pneumonia $(\mathrm{KpC} 4)$ infecting maize and mice. Journal of Integrative Agriculture. 15(7):1510-1520.

[8] Nodia Wohwend, Andrea Endimiani, Thierry Francey and Vincet Perreten. 2015. Third generation cephalosporin resistant klebsiella pneumonia isolates from humans and companion animals in Switzerland: spread of a DHA-producing sequence type 11 clone in a veterinary setting. Antimicrob Agents Chemother. 29:2949-2955.

[9] Podschun, R and Ullmann, U. 1998. Klebsiella sp. as Nosocomial pathogens: Epidemiology, Taxonomy, Typing methods and pathogenicity factor. Clinical Microbiology Reviews. 11(4):584-603.

[10] Razmyar, J and A.H. Zamani. 2016. An outbreak of yolk sac infection and dead-in-shell mortality in common canary caused by Klebsiella pneumonia. Iran J. Vet. Res. 17(2):141-143.

[11] Robert, D E., Mc Clain H M., Hansen D S., Currin P., Howerth E W.2000. An outbreak of Klebsiella pneumonia infection in dogs with severe enteritis and septicaemia. Journal. Vet. Diagn. Invest. 12(2):168-173

[12] Thea Brabb., Denise Newsome., Andrew Burich and Martha Hanes. 2012. The laboratory rabbits, Guinea pigs, Hamster and other rodents. Academic Press. 637-683pp.

[13] Wen Liang $\mathrm{Yu}$ and Yin Ching Chuang. 2013. Clinical features, diagnosis and treatment of Klebsiella pneumonia infection. Up to Date. Dec 18. 2013. 